\title{
Construction of a Non-Trivial Planar Field Theory with Ultraviolet Stable Fixed Point
}

\author{
Giovanni Felder \\ Theoretische Physik, ETH-Hönggerberg, CH-8093 Zürich, Switzerland
}

\begin{abstract}
We study a $\phi_{4}^{4}$ planar euclidean quantum field theory with propagator $1 / p^{2-\varepsilon / 2}, \varepsilon>0$. With the help of the tree expansion of Gallavotti and Nicolò [1], this non-renormalizable theory is shown to have a non-trivial ultraviolet-stable fixed point at negative coupling constant. The vicinity of the fixed point is discussed.
\end{abstract}

\section{Introduction}

We consider a $\phi_{4}^{4}$ theory with propagator $1 / p^{2-\varepsilon / 2}, \varepsilon>0$. The coupling constant has a mass dimension $\varepsilon$ making the theory non-renormalizable and very similar to a $\phi_{4+\varepsilon}^{4}$ model. In particular, one expects to find an ultraviolet stable fixed point at negative coupling. For the planar approximation (i.e. if we keep only the planar Feynman graphs in perturbation theory) we prove that this fixed point does exist, yielding a scale invariant field theory. An expansion about this fixed point provides then a two-parameter family of continuum theories.

The method we used is the tree expansion of Gallavotti and Nicolò [1] which is based on the ideas of Wilson [2] (see also [3], where a similar way of implementing Wilson's ideas in perturbation theory is presented). The tree expansion is an expansion in powers of the running coupling constants on all scales, which is finite order by order (and convergent in planar theories) even if the theory is nonrenormalizable [4]. The running coupling constants are related to one another by recursion relations (the flow equations) and the problem is reduced to the simpler one of finding a solution to the flow equations which is in the convergence domain of the tree expansion. If $\varepsilon$ is small a two-parameter family of solutions is shown to exist in the planar diagram approximation. This gives a rigorous meaning to the $\varepsilon$-expansion [2] for the $N \rightarrow \infty$ limit of an $N \times N$ matrix model with $\operatorname{tr} \phi^{4}$ interaction.

The paper which is largely self-contained is organized as follows: Section 2 is a summary of the methods in [1] applied to the present model: the tree expansion of the effective potentials and the flow equations are formulas (2.14), (2.19). The 
convergence of the tree expansion (Theorem 3.1) is proven in Sect. 3 (see also [4] where similar results are presented for general scalar theories with $\varepsilon=0$ ). In Sect. 4 the fixed-point solution is constructed and in Sect. 5 an expansion around the fixed point is shown to yield a two-parameter family of solutions of the flow equations.

For background on the tree expansion see [1]; for a discussion of planar theories see [5b]. Results on 4-dimensional planar theories are presented in $[5,6]$.

\section{The Tree Expansion and the Beta Functional}

This section is a short review of the tree expansion of Gallavotti and Nicolò [1] applied to our example. We define the main concepts in the full $\phi^{4}$-theory: the planar approximation will be introduced in the computation of truncated expectations.

We consider a perturbation of a free field $\phi$, a Gaussian random field with mean zero and covariance

$$
C(x, y)=\frac{1}{(2 \pi)^{4}} \int \frac{1}{p^{2-\varepsilon / 2}} e^{i p(x-y)} d^{4} p, \quad \varepsilon>0 \quad \text { small }
$$

in four-dimensional euclidean space-time. We introduce a scale decomposition of $\phi$ by writing

$$
\phi=\sum_{j=-\infty}^{+\infty} \phi^{(j)}
$$

where $\phi^{(j)}$ are independent Gaussian fields with mean zero and covariance (in momentum space)

$$
\hat{C}^{(j)}(p)=\int_{\gamma^{-2 j}}^{\gamma^{-2(j-1)}} e^{-\alpha p^{2}} \frac{\alpha^{-\varepsilon / 4} d \alpha}{\Gamma(1-\varepsilon / 4)}
$$

and $\gamma>1$ is some fixed scale factor $(\gamma=2$, say). We have the scaling relations

$$
\begin{gathered}
C^{(j)}(x, y)=\gamma^{(2+\varepsilon / 2) j} C^{(0)}\left(\gamma^{j} x, \gamma^{j} y\right) \\
\phi^{j}(\cdot) \underset{\text { distr. }}{=} \phi^{0}\left(\gamma^{j} \cdot\right) \gamma^{(1+\varepsilon / 4) j},
\end{gathered}
$$

and the bounds $\left(\partial^{l}=\prod_{i=1}^{4}\left(\frac{\partial}{\partial x_{\mu}}\right)^{l_{\mu}},|l|=\sum_{\mu} l_{\mu}\right)$,

$$
\left|\partial C^{(j)}(0, x)\right| \leqq C_{1}(l) \gamma^{\left(2+\frac{\varepsilon}{2}+|l|\right) j} e^{-C_{2} \gamma^{j}|x|}
$$

where $C_{1}(l)$ and $C_{2}$ can be chosen independent of $\varepsilon$ in some range, say $-1 \leqq \varepsilon \leqq 1$.

The model is defined by introducing a cut-off on scale $\gamma^{N}$ and taking as bare interaction the potential

$$
\begin{aligned}
V^{(N)} & =\gamma^{-(\varepsilon / 2) N} \lambda_{2^{\prime}}(N) \int:\left(\partial \phi^{\leqq N}\right)^{2}:+\gamma^{(2-\varepsilon / 2) N} \lambda_{2}(N) \int:\left(\phi^{\leqq N}\right)^{2}:+\gamma^{-\varepsilon N} \lambda_{4}(N) \int:\left(\phi^{\leqq N}\right)^{4}: \\
& \equiv \sum_{\alpha=2^{\prime}, 2,4} \gamma^{-\sigma(\alpha) N} \lambda_{\alpha}(N) \mathcal{O}_{\alpha}\left(\phi^{\leqq N}\right),
\end{aligned}
$$

where $\phi^{\leqq N}=\sum_{j=-\infty}^{N} \phi^{j}$ is the cut-off free field with covariance $C^{(\leqq N)}=\sum_{j=-\infty}^{N} C^{(j)}$. 
By the recursive definition

$$
e^{-V^{(k)}(\phi \leqq k)}=E_{k+1} e^{-V^{(k+1)}(\phi \leqq k+1)}, \quad k=-\infty, \ldots, N-1,
$$

where $E_{k+1}$ denotes expectations with respect to $\phi^{(k+1)}$, we introduce a sequence $\left\{V^{(k)}\left(\phi^{(\leqq k)}\right)\right\}_{k=-\infty}^{N}$ of effective potentials, which can be formally expanded in powers of the fields

$$
V^{(k)}\left(\phi^{(\leqq k)}\right)=\sum_{m=0}^{\infty} \int V_{m}^{(k)}\left(x_{1}, \ldots, x_{m}\right): \phi^{\leqq k}\left(x_{1}\right) \ldots \phi^{\leqq k}\left(x_{m}\right):
$$

for some euclidean invariant distributions $V_{m}^{(k)}$.

For the planar theory we identify the effective potentials with the sequences $\left\{V_{m}^{(k)}\right\}_{m=0}^{\infty}$ from which all Schwinger functions can be computed (see [1b]).

The (dimensionless) running coupling constants (or form factors) $\lambda_{\alpha}(k)$ are defined by

$$
\begin{aligned}
\gamma^{-\varepsilon k} \lambda_{4}(k) & =\int V_{4}^{(k)}\left(0, x_{2}, x_{3}, x_{4}\right) d x_{2} d x_{3} d x_{4}=\hat{V}_{4}(0), \\
\gamma^{-(2-\varepsilon / 2) k} \lambda_{2}(k) & =\int V_{2}^{(k)}(0, x) d x=\hat{V}_{2}(0), \\
\gamma^{-(\varepsilon / 2) k} \lambda_{2^{\prime}}(k) & =\frac{1}{8} \int x^{2} V_{2}^{(k)}(0, x) d x=\left.\frac{\partial}{\partial p^{2}} \hat{V}_{2}\left(p^{2}\right)\right|_{p^{2}=0},
\end{aligned}
$$

and we may decompose the effective potential in the following way:

$$
\begin{aligned}
V^{(k)}\left(\phi^{\leqq k}\right) & =\sum_{\alpha} \gamma^{-\sigma(\alpha) k} \lambda_{\alpha}(k) \mathcal{O}_{\alpha}\left(\phi^{\leqq k}\right)+\sum_{m} \int V_{\mathrm{ren}, m}^{(k)}\left(x_{1}, \ldots, x_{m}\right): \phi_{\left(\bar{x}_{1}\right)}^{\leq k} \ldots \phi \phi_{\left(\bar{x}_{m}\right)} k \\
& \equiv L_{k} V^{(k)}+\left(1-L_{k}\right) V^{(k)},
\end{aligned}
$$

where the linear localization (or Taylor) operator $L_{k}$ acts on Wick polynomials in $\phi^{\leqq k}$ with euclidean invariant kernels $V$ as

$$
\begin{gathered}
L_{k} \int V\left(x_{1}, \ldots, x_{m}\right): \phi^{\leqq k}\left(x_{1}\right) \ldots \phi^{\leqq k}\left(x_{m}\right):=0, \quad \text { if } m>4, \\
L_{k} \int V\left(x_{1}, \ldots, x_{4}\right): \phi^{\leqq k}\left(x_{1}\right) \ldots \phi^{\leqq k}\left(x_{4}\right):=\int V\left(x_{1}, \ldots, x_{4}\right):\left(\phi^{\leqq k}\left(x_{1}\right)\right)^{4}:, \\
L_{k} \int V\left(x_{1}, x_{2}\right): \phi^{\leqq k}\left(x_{1}\right) \phi^{\leqq k}\left(x_{2}\right): \\
=\int V\left(x_{1}, x_{2}\right): \phi^{\leqq k}\left(x_{1}\right):\left(\phi^{\leqq k}\left(x_{1}\right)+\frac{1}{8}\left(x_{2}-x_{1}\right)^{2} \Delta \phi\left(x_{1}\right)\right) .
\end{gathered}
$$

[To be precise, since the Wick monomials in the fields are not given a meaning in the planar theory, we should replace in the definition $(2.11): \phi\left(x_{1}\right) \ldots \phi\left(x_{m}\right):$ by $f_{1}\left(x_{1}\right) \ldots f_{m}\left(x_{m}\right)$, for the test functions $\left.f_{1}, \ldots, f_{m}.\right]$

The cumulant expansion (for the irrelevant terms)

$$
V^{(k)}=\sum_{\alpha} \gamma^{-\sigma(\alpha) k} \lambda_{\alpha}(k) \mathcal{O}_{\alpha}\left(\phi^{\leqq k}\right)+\left(1-L_{k}\right) \sum_{s=1}^{\infty} \frac{(-)^{s-1}}{s !} E_{k+1}^{T}\left(V^{(k+1)}, \ldots, V^{(k+1)}\right)
$$

generates by iteration terms of the type $(k<h<j)$

$$
\begin{aligned}
& \gamma^{-2 \varepsilon j-(\varepsilon / 2) h} \lambda_{4}(j)^{2} \lambda_{2^{\prime}}(h) E_{k+1} E_{k+2} \ldots E_{h-1}\left(1-L_{h-1}\right) \\
& \quad \cdot E_{h}^{T}\left(E_{h+1} \ldots E_{j-1}\left(1-L_{j-1}\right) E_{j}^{T}\left(\int: \phi^{4}:, \int: \phi^{4}:\right), \int:(\partial \phi)^{2}:\right)
\end{aligned}
$$


Fig. 1. A simple tree

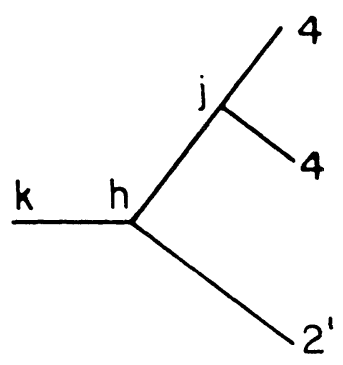

Fig. 2. A general tree

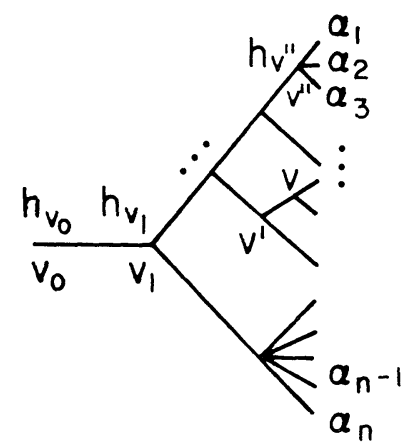

[we have used $E^{T}(\cdot)=E(\cdot), E_{k} L_{k}=L_{k-1} E_{k}{ }^{1}, L_{k}^{2}=L_{k}$ ] which can be efficiently labelled by trees $\theta$. For instance the term (2.13) is labelled by the tree depicted in Fig. 1.

In general, the tree expansion is written as

$$
V^{(k)}=L_{k} V^{(k)}+\sum_{\substack{\theta, \mathbf{h}, \boldsymbol{\alpha} \\ h_{V_{0}}=k}} \frac{1}{n(\theta)} V_{\text {ren }}(\theta, \mathbf{h}, \boldsymbol{\alpha}) \prod_{i \text { end points of } \theta} \lambda_{\alpha_{\imath}}\left(h_{i}\right),
$$

where, if $\theta$ is a tree, $\mathbf{h}=\left(h_{V}\right)_{V \in \theta}$ are integer labels of the branching points $V$ of $\theta$ with $h_{V^{\prime}}<h_{V^{\prime \prime}}$ if $V^{\prime}<V^{\prime \prime}$ in the ordering of $\theta$. The endpoints $i$ of $\theta$ are given labels $\alpha_{i} \in\left\{2^{\prime}, 2,4\right\}$, and $h_{i}$ is the label assigned to the branching point to which the endpoint $i$ is connected. The root $V_{0}$ is given the frequency label $k$ of the effective potential, and the sum in (2.14) is over all possible non-trivial trees, frequency assignments $\mathbf{h}$, and endpoint labels $\boldsymbol{\alpha}$. The combinatorial factor is

$$
n(\theta)=\prod_{V \in \theta} s_{V} !,
$$

where $s_{V}$ is the number of subtrees into which $\theta$ branches at $V$. Figure 2 shows a general tree with root $V_{0}$ and first branching point $V_{1}$. The tree coefficients $V(\theta, \mathbf{h}, \boldsymbol{\alpha})$ can be computed by the following recursive formulas: if $\theta=\theta_{0}$ is the trivial tree (consisting only of the trunk)

$$
V_{\text {ren }}\left(\theta_{0}, h_{V_{0}}, \alpha\right)=\gamma^{-\sigma(\alpha) h_{V_{0}} \mathcal{O}_{\alpha}\left(\phi^{\leqq} h_{V_{0}}\right),}
$$

1 This follows from $E_{k}: \phi^{\leqq k}\left(x_{1}\right) \ldots \phi^{\leqq k}\left(x_{m}\right):=: \phi^{\leqq k-1}\left(x_{1}\right) \ldots \phi^{\leqq k-1}\left(x_{m}\right)$ : 
and if $\theta$ branches at the first branching point $V_{1}$ into trees $\theta_{1}, \ldots, \theta_{s}$,

$$
\begin{aligned}
V_{\mathrm{ren}}(\theta, \mathbf{h}, \boldsymbol{\alpha})= & E_{h_{V_{0}}+1} \ldots E_{h_{V_{1}}-1}\left(1-L_{h_{V_{1}}-1}\right) \\
& \cdot E_{h_{V_{1}}}^{T}\left(V_{\mathrm{ren}}\left(\theta_{1}, \mathbf{h}_{1}, \boldsymbol{\alpha}_{1}\right), \ldots, V_{\mathrm{ren}}\left(\theta_{s}, \mathbf{h}_{\mathrm{s}}, \boldsymbol{\alpha}_{\mathrm{s}}\right),\right.
\end{aligned}
$$

$\mathbf{h}_{r}, \boldsymbol{\alpha}_{r}$ being the restriction of $\mathbf{h}, \boldsymbol{\alpha}$ to $\theta_{r}, r=1, \ldots, s$.

Note that (2.14) is an expansion in powers of all running coupling constants $\lambda_{\alpha}(h)$ and that the only cut-off dependence arises from the fact that the sum in (2.14) is restricted to $h_{V} \leqq N, V \in \theta$, so that the cut-off can be removed simply by ignoring this restriction.

If we act with $L_{k}$ on (2.7) in cumulant expansion

$$
V^{(k)}=\sum_{s=1}^{\infty} \frac{(-)^{s-1}}{s !} E_{k+1}^{T}\left(V^{(k+1)}, \ldots, V^{(k+1)}\right),
$$

and insert (2.14) on the right-hand side, we recover a recursion relation between the running coupling constants (the flow equations)

$$
\lambda_{\alpha}(k)=\gamma^{-\sigma(\alpha)} \lambda_{\alpha}(k+1)+\sum_{\substack{\theta, \mathbf{h}, \boldsymbol{\alpha} \\ h_{V_{0}}=k \\ h_{V_{1}}=k+1}} \frac{1}{n(\theta)} \beta_{\alpha}(\theta, \mathbf{h}, \boldsymbol{\alpha}) \prod_{i \text { endpoints }} \lambda_{\alpha_{2}}\left(h_{i}\right),
$$

where, again, $\sigma\left(2^{\prime}\right)=\varepsilon / 2, \sigma(2)=-2+\varepsilon / 2, \sigma(4)=\varepsilon$ are the dimensions of $\lambda_{2^{\prime}}, \lambda_{2}, \lambda_{4}$ and the sum is over all nontrivial trees. For a tree $\theta$ branching at $V_{1}$ into $\theta_{1}, \ldots, \theta_{s}$, the beta functional [1] coefficient $\beta_{\alpha}(\theta, \mathbf{h}, \boldsymbol{\alpha})$ is defined by

$$
\sum_{\alpha} \gamma^{-\sigma(\alpha) k} \beta_{\alpha}(\theta, \mathbf{h}, \boldsymbol{\alpha}) \mathcal{O}_{\alpha}(\phi \leqq k)=L_{k} E_{k+1}^{T}\left(V_{\mathrm{ren}}\left(\theta_{1}, \mathbf{h}_{1}, \boldsymbol{\alpha}_{1}\right), \ldots, V_{\mathrm{ren}}\left(\theta_{s}, \mathbf{h}_{s}, \boldsymbol{\alpha}_{s}\right)\right) .
$$

Again, the cut-off is removed by summing over $\mathbf{h}$ in (2.19), without restriction.

If we expand $V_{\mathrm{ren}}(\theta, \mathbf{h}, \boldsymbol{\alpha})$ in powers of the fields,

$$
V_{\mathrm{ren}}(\theta, \mathbf{h}, \boldsymbol{\alpha})=\sum_{m} \int V_{\mathrm{ren}}\left(\theta, \mathbf{h}, \boldsymbol{\alpha} ; x_{1}, \ldots, x_{m}\right): \phi^{\leqq h_{V_{0}}}\left(x_{1}\right) \ldots \phi^{\leqq h_{V_{0}}}\left(x_{m}\right):,
$$

we can compute the kernels $V_{m}^{(k)}$ as sum over Feynman graphs, of which we keep only the planar ones:

$$
V_{\text {ren }}\left(\theta, \mathbf{h}, \boldsymbol{\alpha} ; x_{1}, \ldots, x_{m}\right)=\sum_{G \text { comp. with } \theta, \boldsymbol{\alpha}} V_{\text {ren, } G}\left(\theta, \mathbf{h}, \boldsymbol{\alpha} ; x_{1}, \ldots, x_{m}\right),
$$

where the sum is over all labelled planar Feynman graphs with $m$ external lines and $n$ vertices, if $\theta$ has $n$ endpoints, which are compatible with $\theta, \alpha$. A graph $G$ is compatible with $\theta, \alpha$ if the following two conditions are satisfied:

(a) For $i=1, \ldots, n$, the $i^{\text {th }}$ vertex is an $\mathcal{O}_{\alpha_{i}}$ insertion, i.e. it has coordination number 4 if $\alpha_{i}=4$ and 2 if $\alpha=2,2^{\prime}$.

(b) $G$ has two types of internal lines: hard lines (coming from the truncated expectations $E_{h}^{T}$ ) and soft lines (coming from Wick reordering). If we draw a bubble around the vertices corresponding to the endpoints of the subtrees of $\theta$, then the hard lines of $G$ should connect all bubbles contained in any bubble.

Figure 3 shows a graph which is compatible with the tree of Fig. 1; the $\partial$ 's recall that the 3 rd vertex is a $\int:(\partial \phi)^{2}$ : insertion. 


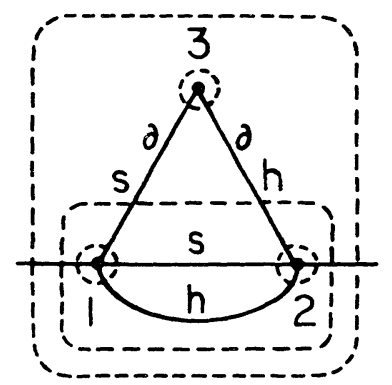

Fig. 3. A Feynman graph compatible with the tree of Fig. $1, h=$ hard, $s=$ soft. The $\partial$ 's symbolize the gradients that act on the propagators

\section{Convergence of the Tree Expansion for the Planar Theory}

The effective potentials of the planar theory are formal power series in the fields

$$
V^{(k)}=\sum_{m=0}^{\infty} \int V_{m}^{(k)}\left(x_{1}, \ldots, x_{m}\right): \phi^{\leqq k}\left(x_{1}\right) \ldots \phi^{\leqq k}\left(x_{m}\right): d x_{1} \ldots d x_{m}
$$

where the kernels $V_{m}^{(k)}$ are symmetric, euclidean invariant distributions. The effective potentials are brought to the form (3.1) by a formal partial integration of a sum of Wick monomials in $\phi^{\leqq k}$ and $\partial \phi^{\leqq}$. We assume therefore that $V_{m}^{(k)} \in \mathscr{V}_{2}\left(\mathbb{R}^{4 m}\right)$, where

$$
\begin{aligned}
\mathscr{V}_{l}\left(\mathbb{R}^{4 m}\right)= & \left\{V \in \mathscr{S}^{\prime}\left(\mathbb{R}^{4 m}\right) \mid V\left(x_{1}, \ldots, x_{m}\right)=\sum_{\substack{\left\{l_{i}, k_{i}, j_{i} l_{l} \leq=1 \\
0 \leqq l_{l} \leqq l \\
1 \leqq j_{i}, k_{l} \leqq m\right.}} \bar{V}_{\left\{l_{i}, k_{i}, j_{i}\right\}}\left(x_{1} \ldots x_{m}\right)\right. \\
& \left.\cdot \prod_{i=1}^{s} \partial^{l_{l}} \delta\left(x_{j_{i}}-x_{k_{i}}\right), \bar{V}_{\left\{l_{i}, k_{i}, j_{i}\right\}} \in L_{\text {loc }}^{1}\left(\mathbb{R}^{4 m}\right)\right\} .
\end{aligned}
$$

The kernels $V_{m}^{(k)}$ can be computed in tree expansion (2.14) as a power series in the running coupling constants $\lambda_{\alpha}(h)$, which verify the recursion relation (2.19). The aim of this section is to prove that $(2.14),(2.19)$ are convergent expansions for small $\lambda_{\alpha}$ 's.

The dimension of a Wick monomial of degree $m$, with $l$ gradients is defined by

$$
\sigma(m, l)=-4+(1-\varepsilon / 4) m+l .
$$

The result of this section is the following:

Theorem 3.1. (i) Let $f_{1}, \ldots, f_{m}$ be $\mathscr{S}\left(\mathbb{R}^{4}\right)$-test functions. Then for $|\varepsilon| \leqq \varepsilon_{0}$ and $\theta$ a nontrivial tree,

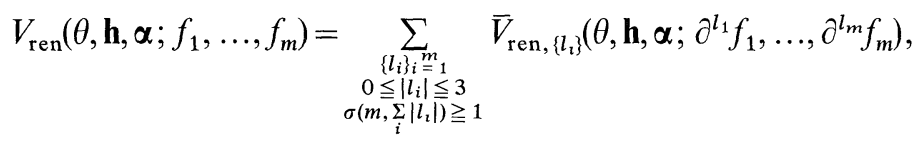


where $\bar{V}_{\text {ren, } 1} \in \mathscr{V}_{0}\left(\mathbb{R}^{4 m}\right)$ and

$$
\begin{aligned}
& \int_{\Delta_{2} \times \ldots \times \Delta_{m}}\left|\bar{V}_{\text {ren },\left\{l_{i}\right\}}\left(0, x_{2}, \ldots, x_{m}\right)\right| d x_{2} \ldots d x_{m} \\
& \leqq C_{0}^{n} n(\theta) e^{\left.-\frac{c_{2}}{2} \gamma^{h_{V_{0}} \operatorname{dist}\left(0, \Delta_{2}, \ldots, \Delta_{m}\right)} \gamma^{-\sigma\left(m, \Sigma_{i}\left|l_{i}\right|\right.}\right)} \prod_{\substack{V \in \theta \\
V>V_{0}}} \gamma^{-\frac{1}{2}\left(h_{V}-h_{V^{\prime}}\right)} .
\end{aligned}
$$

(ii) For $|\varepsilon| \leqq \varepsilon_{0}$,

$$
\left|\beta_{\alpha}(\theta, \mathbf{h}, \boldsymbol{\alpha})\right| \leqq C_{0}^{n} n(\theta) \prod_{\substack{V \in \theta \\ V>V_{1}}} \gamma^{-\frac{1}{2}\left(h_{V}-h_{V^{\prime}}\right)},
$$

where $n \geqq 2$ is the number of endpoints of $\theta ; \Delta_{2} \ldots \Delta_{m}$ are cubes in $\mathbb{R}^{4}$ with side size $\gamma^{-h_{V_{0}}} ; \operatorname{dist}\left(0, \Delta_{2}, \ldots, \Delta_{m}\right)$ is the length of the shortest graph connecting $0, \Delta_{2}, \ldots, \Delta_{m}$; $V_{0}$ is the root of $\theta, V_{1}$ the first branching point, and, for $V \in \theta, V^{\prime}$ is the branching point immediately preceding $V$ (see Fig. 2); $C_{0}=C_{0}\left(\varepsilon_{0}, \gamma\right)$ is a positive constant and $\mathrm{C}_{2}$ is the constant appearing in (2.5).

Discussion. The exponential decay factors $\gamma^{-\frac{1}{2}\left(h_{V}-h_{V^{\prime}}\right)}$ (recall that $h_{V^{\prime}}<h_{V}$ ) allow us to sum over all $\mathbf{h}$. There are at most const ${ }^{n}$ trees $\theta$ with $n$ endpoints with labels $\boldsymbol{\alpha}$. This means that the series (2.14), (2.19) are absolutely convergent if $\|\lambda\|_{\infty}$ $\equiv \sup _{\alpha, h}\left|\lambda_{\alpha}(h)\right|<R$ for some $R>0$, and define thus functions on $\left\{\lambda:\|\lambda\|_{\infty}<R\right\} \equiv B_{R}$ :

$$
\begin{gathered}
V^{(k)}=\sum_{\alpha} \gamma^{-\sigma(\alpha) k} \lambda_{\alpha}(k) \mathcal{O}_{\alpha}\left(\phi^{\leqq k}\right)+V_{\mathrm{ren}}^{(k)}(\lambda), \\
\lambda_{\alpha}(k)=\gamma^{-\sigma(\alpha)} \lambda_{\alpha}(k+1)-\beta_{\alpha}^{(k)}(\lambda) .
\end{gathered}
$$

The scaling relations (2.4) imply the "renormalization group equations"

$$
\begin{gathered}
V_{\text {ren }}^{(k+1)}\left(\gamma^{(1+\varepsilon / 4)} \phi^{\leqq k}(\gamma \cdot) ;{ }^{\sigma} \lambda\right)=V_{\text {ren }}^{(k)}\left(\phi^{\leqq k}(\cdot) ; \lambda\right), \\
\beta_{\alpha}^{(k+1)}\left({ }^{\sigma} \lambda\right)=\beta_{\alpha}^{(k)}(\lambda),
\end{gathered}
$$

where $\sigma$ is the shift operator: $\left({ }^{\sigma} \lambda\right)_{\alpha}(k)=\lambda_{\alpha}(k-1)$. These relations will be needed in Sect. 4.

Proof of the Theorem. The proof is in three steps. First, we prove that the operator $1-L_{k}$ generates irrelevant terms with "good" bounds. The second step is an estimate on Feynman amplitudes and the third is an estimate of the sum over all Feynman graphs compatible with a given tree.

1st Step. For $\bar{V} \in \mathscr{V}_{0}\left(\mathbb{R}^{4 m}\right)$ (i.e. a smooth function with decay at infinity times delta functions), we introduce the weighted norm ( $\bar{V}$ is assumed to be euclidean invariant)

where $\varrho \geqq 0$.

$$
\|\bar{V}\|_{\varrho}=\int\left|\bar{V}\left(x_{1}, \ldots, x_{m}\right)\right| e^{\varrho \operatorname{dist}\left(x_{1}, \ldots, x_{m}\right)} d x_{2} \ldots d x_{m},
$$

Proposition 3.2. Let $V \in \mathscr{V}_{2}\left(\mathbb{R}^{4 m}\right)$ and

$$
\begin{gathered}
\left(1-L_{k}\right) \int V\left(x_{1}, \ldots, x_{m}\right): \phi^{\leqq k}\left(x_{1}\right) \ldots \phi^{\leqq k}\left(x_{m}\right): \\
\quad=\int V_{\mathrm{ren}}\left(x_{1}, \ldots, x_{m}\right): \phi^{\leqq k}\left(x_{1}\right) \ldots \phi^{\leqq k}\left(x_{m}\right): .
\end{gathered}
$$


If $V$ has a zero of order l in momentum space, i.e. if for some $\left\{l_{1}\right\}_{i=1}^{m}$ with $\sum_{i}\left|l_{i}\right|=l$,

$$
V\left(f_{1}, \ldots, f_{m}\right)=\bar{V}\left(\partial^{l_{1}} f_{1}, \ldots, \partial^{l_{m}} f_{m}\right), \quad \bar{V} \in \mathscr{V}_{0}\left(\mathbb{R}^{4 m}\right),
$$

then

(i) if $\sigma(m, l) \geqq 1, V_{\text {ren }}=V$,

(ii) if $\sigma(m, l)<1$, i.e. $(m, l)=(4,0),(2,0),(2,1),(2,2)$,

$$
\begin{gathered}
V_{\text {ren }}\left(f_{1}, \ldots, f_{m}\right)=\sum_{\substack{\left\{l_{i}\right\} \\
\left|l_{i}\right| \leqq 3 \\
\Sigma\left|l_{\imath}\right|=l_{\text {ren }}}} \bar{V}_{\text {ren, }\left\{l_{i}\right\}}\left(\partial^{l_{1}} f_{1}, \ldots, \partial^{l_{m}} f_{m}\right), \quad \bar{V}_{\text {ren },\left\{l_{l}\right\}} \in \mathscr{V}_{0}\left(\mathbb{R}^{4}\right), \\
\left\|\bar{V}_{\text {ren },\left\{l_{i}\right\}}\right\|_{\varrho} \leqq\left(\frac{1}{\varrho}\right)^{l_{\text {ren }}-l}\|\bar{V}\|_{\varrho},
\end{gathered}
$$

where $l_{\text {ren }}$ is the smallest integer $\geqq l$ such that $\sigma\left(m, l_{\text {ren }}\right) \geqq 1$.

Proof. (i) follows from the definition of $L_{k}$. To prove (ii) we discuss separately the four cases:

a) $m=4, l=0, V=\bar{V}$

$$
\begin{gathered}
V_{\text {ren }}\left(f_{1}, \ldots, f_{4}\right)=\bar{V}_{\text {ren }, \lambda}^{(2)}\left(f_{1}, \partial_{\lambda} f_{2}, f_{3}, f_{4}\right)+\bar{V}_{\text {ren }, \lambda}^{(3)}\left(f_{1}, f_{2}, \partial_{\lambda} f_{3}, f_{4}\right) \\
+\bar{V}_{\text {ren }, \lambda}^{(4)}\left(f_{1}, f_{2}, f_{3}, \partial_{\lambda} f_{4}\right), \\
\left\|\bar{V}_{\text {ren }, \lambda}^{(i)}\right\|_{\varrho} \leqq \frac{1}{\varrho}\|V\|_{\varrho} .
\end{gathered}
$$

b) $m=2, l=0, V=\bar{V}$

$$
\begin{gathered}
V_{\text {ren }}\left(f_{1}, f_{2}\right)=\bar{V}_{\text {ren }, \lambda \mu \nu}\left(f_{1}, \partial_{\lambda} \partial_{\mu} \partial_{\nu} f_{2}\right), \\
\left\|\bar{V}_{\text {ren }, \lambda \mu \nu}\right\|_{\varrho} \leqq\left(\frac{1}{\varrho}\right)^{3}\|V\|_{\varrho} .
\end{gathered}
$$

c) $m=2, l=1, V\left(f_{1}, f_{2}\right)=\bar{V}\left(f_{1}, \partial_{\lambda} f_{2}\right)$

$$
\begin{gathered}
V_{\text {ren }}\left(f_{1}, f_{2}\right)=\bar{V}_{\text {ren }, \mu \nu}\left(f_{1}, \partial_{\lambda} \partial_{\mu} \partial_{v} f_{2}\right), \\
\left\|\bar{V}_{\text {ren }, \mu \nu}\right\|_{\varrho} \leqq\left(\frac{1}{\varrho}\right)^{2}\|\bar{V}\|_{\varrho} .
\end{gathered}
$$

d) $m=2, l=2, V\left(f_{1}, f_{2}\right)=\bar{V}\left(f_{1}, \partial_{\lambda} \partial_{\mu} f_{2}\right)$

$$
\begin{gathered}
V_{\text {ren }}\left(f_{1}, f_{2}\right)=\bar{V}_{\text {ren }, v}\left(f_{1}, \partial_{\lambda} \partial_{\mu} \partial_{v} f_{2}\right), \\
\left\|\bar{V}_{\text {ren }, v}\right\|_{\varrho} \leqq \frac{1}{\varrho}\|\bar{V}\|_{\varrho} .
\end{gathered}
$$

The most complicated case is b), which we prove explicitly. The other cases are proven the same way, but are simpler. By the Taylor remainder theorem (with 


$$
\begin{aligned}
& \left.x_{21} \equiv x_{2}-x_{1}\right) \text {, } \\
& V_{\text {ren }}\left(f_{1}, f_{2}\right)=\int V\left(x_{1}, x_{2}\right) f_{1}\left(x_{1}\right) \\
& \text { - }\left\{f_{2}\left(x_{2}\right)-f_{2}\left(x_{1}\right)-x_{21}^{\mu} \partial_{\mu} f_{2}\left(x_{1}\right)-\frac{1}{2} x_{21}^{\mu} x_{21}^{\nu} \partial_{\mu} \partial_{v} f_{2}\left(x_{1}\right)\right\} \\
& =\int_{0}^{1} d t \frac{(1-t)^{2}}{2 !}\left(\frac{d}{d t}\right)^{3} \int V\left(x_{1}, x_{2}\right) f_{1}\left(x_{1}\right) f_{2}\left(t x_{2}+(1-t) x_{1}\right) d x_{1} d x_{2} \\
& =\int_{0}^{1} d t \frac{(1-t)^{2}}{2 !} t^{-7} \\
& \cdot \int V\left(\frac{x_{1}}{t}, \frac{x_{2}}{t}\right) x_{21}^{\lambda} x_{21}^{\mu} x_{21}^{\nu} f_{1}\left(x_{1}\right) \partial_{\lambda} \partial_{\mu} \partial_{\nu} f_{2}\left(x_{1}\right) d x_{1} d x_{2} \text {. }
\end{aligned}
$$

This proves $b$ ) with

$$
\bar{V}_{\text {ren, } \lambda \mu \nu}=\int_{0}^{1} d t \frac{(1-t)^{2}}{2 !} t^{-7} V\left(\frac{x_{1}}{t}, \frac{x_{2}}{t}\right) x_{21}^{\lambda} x_{21}^{\mu} x_{21}^{v} .
$$

The bound is

$$
\begin{aligned}
\left\|\bar{V}_{\text {ren, } \lambda \mu \nu}\right\|_{\varrho} & \leqq \int d x \int_{0}^{1} d t \frac{(1-t)^{2}}{2 !}\left|V(0, x) x^{\lambda} x^{\mu} x^{\nu}\right| e^{\varrho|x| t} \\
& \leqq\left(\frac{1}{\varrho}\right)^{3}\|V\|_{\varrho},
\end{aligned}
$$

where we have used

$$
\int_{0}^{1} d t(1-t)^{2}|x|^{3} e^{\varrho|x| t}=e^{\varrho|x|} \frac{1}{\varrho^{3}} \int_{0}^{\varrho|x|} d u u^{2} e^{-u} \leqq \frac{2 !}{\varrho^{3}} e^{\varrho|x|} .
$$

2nd Step. We want now to estimate inductively tree coefficients $V_{\text {ren }}(\theta, \mathbf{h}, \boldsymbol{\alpha})$ using the recursion relation (2.17). Recalling that $E_{k} L_{k}=L_{k-1} E_{k}$, we can write this relation as

$$
\begin{gathered}
V(\theta, \mathbf{h}, \boldsymbol{\alpha})=E_{h_{V_{0}}} \ldots E_{h_{V_{1}}-1} E_{h_{V_{1}}}^{T}\left(V_{\text {ren }}\left(\theta_{1}, \mathbf{h}_{1}, \boldsymbol{\alpha}_{1}\right), \ldots, V_{\text {ren }}\left(\theta_{s}, \mathbf{h}_{s}, \boldsymbol{\alpha}_{s}\right)\right), \\
V_{\text {ren }}(\theta, \mathbf{h}, \boldsymbol{\alpha})=\left(1-L_{h_{V_{0}}}\right) V(\theta, \mathbf{h}, \boldsymbol{\alpha}),
\end{gathered}
$$

and we focus on the contribution $V_{G}$ of a single graph $G$ compatible with $\theta$, $\alpha$, with subgraphs $G_{1}, \ldots, G_{s}$ corresponding to $\theta_{1}, \ldots, \theta_{s}$. We estimate $V_{G}$ by simple power counting and then use Proposition 3.2 to estimate $V_{\text {ren, } G \text {. }}$

Proposition 3.3. Let $G$ be a graph compatible with $\theta$, $\alpha$. Then, for $|\varepsilon|<\varepsilon_{0}$,

$$
V_{\text {ren, } G}\left(\theta, \mathbf{h}, \boldsymbol{\alpha} ; f_{1}, \ldots, f_{m}\right)=\sum_{\substack{\left\{l_{i}\right\} \\\left|l_{i}\right| \leq 3 \\ \sigma\left(m, \sum_{i}\left|\bar{l}_{i}\right|\right) \geqq 1}} \bar{V}_{\text {ren, },\left\{,\left\{l_{i}\right\}\right.}\left(\theta, \mathbf{h}, \boldsymbol{\alpha} ; \partial^{l_{1}} f_{1}, \ldots, \partial^{l_{m}} f_{m}\right),
$$

where $\bar{V}_{\text {ren, },\left\{l_{i}\right\}} \in \mathscr{V}_{0}\left(\mathbb{R}^{4 m}\right)$ and

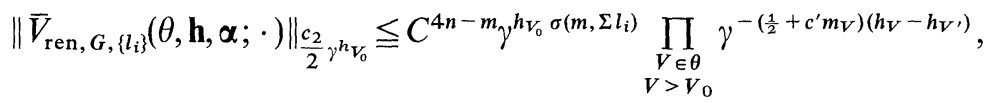



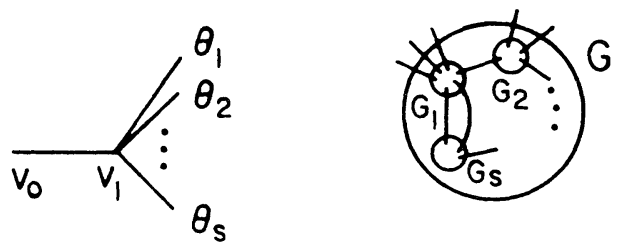

Fig. 4. The setting of the inductive proof: the graph $G$ contributing to the tree $\theta$ has subgraphs $G_{1}, \ldots, G_{s}$ corresponding to the subtrees $\theta_{1}, \ldots, \theta_{s}$

where $n$ is the number of endpoints of $\theta$ (and of vertices of $G$ ) and $m_{V}$ is the number of external lines of the subgraph $G_{V}$ of $G$ corresponding to the subtree $\theta_{V}$ of $\theta$ with first branching point $V . C$ and $c^{\prime}$ are positive constants.

Proof. The proof is inductive. We use (3.19) and compute the contribution of $G$ to $V(\theta, \mathbf{h}, \boldsymbol{\alpha})$ from the contributions of the subgraphs $G_{1}, \ldots, G_{s}$ to $V\left(\theta_{1}, \mathbf{h}_{1}, \boldsymbol{\alpha}_{1}\right), \ldots, V\left(\theta_{s}, \mathbf{h}_{s}, \boldsymbol{\alpha}_{s}\right)$ (see Fig. 4). Some of the $\theta_{i}$ 's are trivial trees. They contribute to the computation of $V_{G}$ a factor $\gamma^{-\sigma\left(\alpha_{i}\right) h_{V_{1}}}$, by (2.16). Assume now inductively that the proposition holds for the non-trivial trees in the list $\theta_{1}, \ldots, \theta_{s}$. Then $V_{G}$ is given by a generalized convolution of the kernels $V_{G_{r}}$ with propagators on scale $h_{V_{1}}$ :

$$
\begin{aligned}
V_{G}\left(\theta, \mathbf{h}, \boldsymbol{\alpha} ; f_{1}, \ldots, f_{m}\right)= & \int V_{\mathrm{ren}, G_{1}} \ldots V_{\mathrm{ren}, G_{s}} \prod_{\substack{\langle i, j\rangle \\
\text { hard }}} C^{\left(h_{V_{1}}\right)}\left(x_{i}, x_{j}\right) \\
& \cdot \prod_{\substack{\langle i, j\rangle \\
\text { soft }}} C^{\left(<h_{V_{1}}\right)}\left(x_{i}, x_{j}\right) \prod_{i=1}^{m} f_{i}\left(x_{i}\right) \prod_{r} d^{4} x_{r} .
\end{aligned}
$$

For the trivial trees $\theta_{r}, V_{\text {ren, } G_{r}}$ is replaced by $\gamma^{-\sigma\left(\alpha_{r}\right) h_{V_{1}}}$ in (2.23). After replacing $V_{\text {ren, } G_{r}}, r=1, \ldots, s$, by $\bar{V}_{\text {ren, } G_{r},\{l\}}$ and integrating over the internal vertices, $V_{G}$ becomes an expression of the form (we suppress the $\theta, \mathbf{h}, \boldsymbol{\alpha}$-dependence):

$$
V_{G}\left(f_{1}, \ldots, f_{m}\right)=\sum_{\substack{\left\{l_{i}\right\} \\\left|l_{i}\right| \leqq 3}} \bar{V}_{G,\left\{l_{i}\right\}}\left(\partial^{l_{1}} f_{1}, \ldots, \partial^{l_{m}} f_{m}\right)
$$

and, to estimate $\left\|V_{G}\right\|_{\frac{1}{2} c_{2} \gamma^{h} V_{1}}$, we note that

$$
\gamma^{h_{V_{1}}} \operatorname{dist} x_{G} \leqq \gamma^{h_{V_{1}}} \sum_{r=1}^{s} \operatorname{dist} x_{G_{r}}+\gamma^{h_{V_{1}}} \sum_{\langle i, j\rangle \in T}\left|x_{i}-x_{j}\right|,
$$

where $x_{G}$ are the points associated with the vertices of $G$ connected to external lines, and $T$ is some tree subgraph of hard lines of $G$ connecting $G_{1}, \ldots, G_{s}$. For the propagators and their gradients we insert the bounds (2.5) (note that we have at most 6 derivatives acting on a propagator):

$$
\begin{aligned}
\left|\partial^{l} C^{(h)}\left(x_{i}, x_{j}\right)\right| & \leqq c_{1}(l) \gamma^{(2+\varepsilon / 2+|l|) h} e^{-c_{2} \gamma^{h}\left|x_{i}-x_{j}\right|}, \\
\left|\partial^{l} C^{(<h)}\left(x_{i}, x_{j}\right)\right| & \leqq \frac{c_{1}(l)}{1-\gamma^{-2}} \gamma^{(2+\varepsilon / 2+|l|) h} .
\end{aligned}
$$


We keep the exponential decay factor $e^{-c_{2} \gamma^{h}\left(x_{i}-x_{j}\right)}$ only for $(i, j) \in T$. This allows us to integrate over the internal vertices. The result is $\left(\right.$ with $\left.c_{1}=\max _{0 \leqq l \leqq 6} \frac{c_{1}(l)}{1-\gamma^{-2}}\right)$

$$
\begin{aligned}
& \left.\left\|\bar{V}_{G,\left\{l_{i}\right)}\right\|_{\frac{1}{2} c_{2} \gamma^{h} V_{1}} \leqq \sum_{\{l(1)\}_{l}, \ldots,\{l(s)\}} \gamma^{[(2+\varepsilon / 4)}\left(\sum_{r=1}^{s} m_{r}-m\right)+\sum_{r=1}^{s}|l(r)|-|l|\right] h^{h} \\
& \text {. } C_{1}^{\sum m_{r}-m} B_{1}^{s-1} \gamma^{-4(s-1) h_{V_{1}}} \prod_{r=1}^{s}\left\|\bar{V}_{\mathrm{ren}, G_{r},\{l(r)}\right\|_{\frac{1}{2} c_{2} \gamma^{h} V_{1}} \\
& \leqq B_{2}^{\sum m_{r}-m} C^{4 n-\Sigma m_{r}} \gamma^{-\sigma(m,|l|) h_{V_{1}}} \prod_{V>V_{1}} \gamma-\left(\frac{1}{2}+C^{\prime} m_{V}\right)\left(h_{V}-h_{V^{\prime}}\right),
\end{aligned}
$$

where $|l| \equiv \sum_{i}\left|l_{i}\right|$ and $B_{1}, B_{2}$ are positive constants. For $\theta_{r}$ trivial, $\left\|\bar{V}_{\text {ren, } G_{r},\{l(r)}\right\| \|_{\frac{c_{2}}{2}} \gamma_{V_{V_{1}}}$ is replaced by $\gamma^{-\sigma\left(\alpha_{r}\right) h_{V_{1}}}$, with same bound. The coefficient $\left(\frac{1}{2}+c^{\prime} m_{V}\right)$ comes from

$$
\sigma\left(m,\left|l^{(r)}\right|\right) \geqq \max \left\{1,-4+(1+\varepsilon / 4) m_{r}\right\} \geqq \frac{1}{2}+c^{\prime} m_{r}, \quad c^{\prime}=c^{\prime}\left(\varepsilon_{0}\right)>0,
$$

and $m_{r}$ is the number of external lines of $G_{r}, r=1, \ldots, s$. We then apply Proposition 3.2 to estimate $\bar{V}_{\text {ren, } G,\left\{l_{i}\right\}}$ :

$$
\begin{aligned}
& \left\|\bar{V}_{\text {ren, } G,\left\{l_{i}^{\prime}\right\}}\right\|_{\frac{1}{2} c_{2} \gamma^{h_{V_{o}}}} \leqq\left\|\bar{V}_{\text {ren, } G,\left\{l_{i}^{\prime}\right\}}\right\|_{\frac{1}{2} c_{2} \gamma^{h} V_{1}} \\
& \leqq \sum_{\left\{l_{i}\right\}} \gamma^{-h_{V_{1}}\left(\left|l^{\prime}\right|-|l|\right)}\left\|\bar{V}_{G,\left\{l_{i}\right\}}\right\|_{\frac{1}{2} c_{2} \gamma^{h_{V_{1}}}} \\
& \leqq C^{4 n-m} \gamma^{-h_{V_{1}} \sigma\left(m,\left|l^{\prime}\right|\right)} \prod_{V>V_{1}} \gamma^{-\left(\frac{1}{2}+c^{\prime} m_{V}\right)\left(h_{V}-h_{V^{\prime}}\right)} \\
& \leqq C^{4 n-m} \gamma^{\left.-h_{V_{0}} \sigma\left(m, \mid l^{\prime}\right)\right)} \prod_{V>V_{0}} \gamma^{-\left(\frac{1}{2}+c^{\prime} m_{V}\right)\left(h_{V}-h_{V^{\prime}}\right)} .
\end{aligned}
$$

Here $\bar{V}_{G,\left\{l_{i}\right\}}$ are the terms in (3.24) that give $\bar{V}_{\text {ren, } G,\left\{l_{i}^{\prime}\right\}}$ under the action of $\left(1-L_{h_{V_{0}}}\right)$. Note that by Proposition 3.2, $\sigma(m,|l|) \geqq 1$. Proposition 3.3 is thus proven, with $C$ large compared to $B_{2}$.

3rd Step. It is well-known [7] that there exist at most const ${ }^{n}$ planar unlabelled Feynman graphs with $n$ vertices. A simple combinatorial argument (see [1b, Appendix F]) shows that an unlabelled graph with $n$ vertices can be labelled in at most $C_{\delta}^{n} n(\theta) \exp \delta \sum_{V \in \theta} m_{V}$ ways to be compatible with a tree $\theta$ such that, for all $V \in \theta$, the subgraph $G_{V}$ corresponding to $V$ has $m_{V}$ external lines; $\delta$ can be chosen as small as desired. We can thus sum over all graphs the bounds of Proposition 3.3:

$$
\left\|\bar{V}_{\text {ren, }\left\{l_{i}\right\}}(\theta, \mathbf{h}, \boldsymbol{\alpha} ; \cdot)\right\|_{\frac{1}{2} c_{2} \gamma^{h_{V_{0}}}} \leqq C_{0}^{n} \gamma^{h_{V_{0}} \sigma\left(m, \Sigma\left|l_{i}\right|\right)} \prod_{V>V_{0}} \gamma^{-\frac{1}{2}\left(h_{V}-h_{V^{\prime}}\right)}
$$

with $\delta=c^{\prime} \ln \gamma, C_{0}=$ const $C_{\delta} C^{4}$. The claim of the theorem follows by restricting the integration in \|\| to the domain $\Delta_{2} \times \ldots \times \Delta_{m}$.

\section{Construction of the Fixed Point}

The construction of a planar field theory is now simple: it is sufficient to find a solution, $\lambda$, of (3.8), with $\lambda \in B_{R}$, and the effective potentials are then given by (3.7). 
One could think of finding a solution of (3.8) by expressing all $\lambda_{\alpha}(k)$ as power series in $\lambda_{\alpha}\left(k_{0}\right)$ with e.g. $k_{0}=0$ by iterating (3.8). This fails because the expansion in the renormalized coupling constants is plagued with the ultraviolet divergences of a non-renormalizable field theory (see [4] for a discussion of this point).

But (3.8) has a fixed-point solution as one can see by choosing the ansatz

$$
\lambda_{\alpha}(k)=\lambda_{\alpha}^{*}, \quad k=-\infty, \ldots,+\infty,
$$

and solving the equation $\left[\right.$ with $\left.\beta_{\alpha}^{(k)}\left(\lambda_{\alpha}(k)=\lambda^{*}\right) \equiv \bar{\beta}_{\alpha}\left(\lambda^{*}\right)\right]$

$$
\lambda_{\alpha}^{*}=\gamma^{-\sigma(\alpha)} \lambda_{\alpha}^{*}-\bar{\beta}_{\alpha}\left(\lambda^{*}\right)
$$

where $\bar{\beta}_{\alpha}$ is an analytic function of the three variables $\lambda_{2}^{*}, \lambda_{2}^{*}, \lambda_{4}^{*}$ for $\left|\lambda_{\alpha}^{*}\right| \leqq R$. The scaling relation (3.10) implies that, with this ansatz, all (3.8) are satisfies if (4.2) is, and that $\bar{\beta}_{\alpha}$ does not depend on $k$.

The functions $\bar{\beta}_{\alpha}$ can be computed explicitly up to second order:

$$
\begin{aligned}
& \bar{\beta}_{4}(\lambda)=\beta_{444} \lambda_{4}^{2}+\beta_{424} \lambda_{2} \lambda_{4}+O\left(\lambda^{3}\right) \\
& \bar{\beta}_{2}(\lambda)=\beta_{244} \lambda_{4}^{2}+\beta_{224} \lambda_{2} \lambda_{4}+\beta_{22^{\prime} 4} \lambda_{2^{\prime}} \lambda_{4}+\beta_{222} \lambda_{2}^{2}+O\left(\lambda^{3}\right), \\
& \bar{\beta}_{2^{\prime}}(\lambda)=\beta_{2^{\prime} 44} \lambda_{4}^{2}+\beta_{2^{\prime} 22} \lambda_{2}^{2}+\beta_{2^{\prime} 22^{\prime}} \lambda_{2} \lambda_{2^{\prime}}+O\left(\lambda^{3}\right)
\end{aligned}
$$

and the coefficients coincide with the second order coefficients of the $\beta$ and $\gamma$ functions of the physics literature.

We solve (4.2) by the analytic implicit function theorem: we define $\bar{\varepsilon}=1-\gamma^{-\varepsilon}$ and write (4.2) as

$$
\begin{aligned}
-\bar{\varepsilon} \lambda_{4}^{*} & =\bar{\beta}_{4}\left(\lambda_{4}^{*}, \lambda_{2}^{*}, \lambda_{2^{\prime}}^{*}\right), \\
\left(\gamma^{2}-1\right) \lambda_{2}^{*} & =\bar{\beta}_{2}\left(\lambda_{4}^{*}, \lambda_{2}^{*}, \lambda_{2^{\prime}}^{*}\right), \\
-\bar{\varepsilon}\left(1+\gamma^{\varepsilon / 2}\right)^{-1} \lambda_{2^{\prime}}^{*} & =\bar{\beta}_{2^{\prime}}\left(\lambda_{4}^{*}, \lambda_{2}^{*}, \lambda_{2^{\prime}}^{*}\right), \quad\left|\lambda_{4}^{*}\right|,\left|\lambda_{2}^{*}\right|,\left|\lambda_{2^{\prime}}^{*}\right|<R,
\end{aligned}
$$

which can be solved in a convergent $\bar{\varepsilon}$ expansion, if $\varepsilon$ (and thus $\bar{\varepsilon}$ ) is small enough: the non-trivial solution

$$
\begin{aligned}
& \lambda_{4}^{*}=-\bar{\varepsilon} \beta_{444}^{-1}+O\left(\bar{\varepsilon}^{2}\right) \\
& \lambda_{2}^{*}=\bar{\varepsilon}^{2} \frac{1}{\gamma^{2}-1} \beta_{444}^{-2}\left(\beta_{244}+2 \beta_{22^{\prime} 4} \beta_{2^{\prime} 44} \beta_{444}^{-1}\right)+O\left(\bar{\varepsilon}^{3}\right), \\
& \lambda_{2^{\prime}}^{*}=-2 \bar{\varepsilon} \beta_{2^{\prime} 44} \beta_{444}^{-1}+O\left(\bar{\varepsilon}^{2}\right),
\end{aligned}
$$

correspond to a sequence of effective potentials via (3.7)

$$
V^{*(k)}=\sum_{\alpha} \gamma^{-\sigma(\alpha) k} \lambda_{\alpha}^{*} \mathcal{O}_{\alpha}\left(\phi^{\leqq k}\right)+V_{\mathrm{ren}}^{*(k)} \quad\left(\lambda_{\alpha}(k)=\lambda_{\alpha}^{*}\right)
$$

with the scale invariance property (3.9)

$$
V^{*(k)}\left(\phi^{\leqq k}\right)=V^{*(0)}\left(\gamma^{-(1+\varepsilon / 4)} \phi^{\leqq k}\left(\gamma^{-k} \cdot\right)\right) .
$$

The details of the application of the implicit function theorem for the solution of (4.4) are contained in the Appendix. 


\section{The Vicinity of the Fixed Point}

In Sect. 4 we have constructed one single theory, the fixed point (scale invariant) theory. In this section we show that one can construct a two parameter family of solutions $\left\{\lambda_{\alpha}(k)\right\}$ of the flow equations with positive $k$, which yields a two parameter family of effective potentials on all scales $k \geqq 0$. These solutions are such that $\lambda_{\alpha}(0)$ is very close to the fixed point and $\lambda_{\alpha}(k)$ approaches the fixed point exponentially fast. The central idea in the proof is that if one expands around the non-trivial fixed point there are two relevant directions and no marginal ones, so that the situation is very similar to a superrenormalizable theory!

The strategy is the following: we first expand the flow equations around the fixed point and diagonalize the linearization of the beta functional at the fixed point to first order in $\varepsilon$. Then the contraction principle yields a two parameter family of solutions.

We first simplify the notation: the (convergent) power series (2.19) can be written as

$$
\begin{aligned}
\lambda_{\alpha}(k) & =\gamma^{-\sigma(\alpha)} \lambda_{\alpha}(k+1)-\beta_{\alpha}^{(k)}(\lambda), \\
\beta_{\alpha}^{(k)}(\lambda) & =\sum_{\substack{\mathbf{n} \\
|\mathbf{n}| \geqq 2}} \beta_{\alpha, \mathbf{n}}^{(k)} \lambda^{\mathbf{n}},
\end{aligned}
$$

where $\mathbf{n}=\left\{n_{\alpha}(k)\right\}_{k \in \mathbb{Z}, \alpha=2,4,2^{\prime}}, \lambda^{\mathbf{n}}=\prod_{\alpha, k} \lambda_{\alpha}(k)^{n_{\alpha}(k)}$ and the sum is over all multiindices n with $|\mathbf{n}| \equiv \sum_{\alpha, k} n_{\alpha}(k)<\infty$. It then follows easily from (3.6) that

$$
\begin{gathered}
\beta_{\alpha, \mathbf{n}}^{(k)}=0, \quad \text { if } \quad h_{\min }(\mathbf{n})<k, \\
\left|\beta_{\alpha, \mathbf{n}}^{(k)}\right| \leqq C^{|\mathbf{n}|} \varrho^{-\left(h_{\max }(\mathbf{n})-k\right)}
\end{gathered}
$$

for some ( $\varepsilon$-independent) constants $C>0, \varrho>1$, where

$$
\begin{aligned}
& h_{\max }(\mathbf{n})=\max \left\{h \mid n_{\alpha}(h) \neq 0 \text { for some } \alpha\right\}, \\
& h_{\min }(\mathbf{n})=\min \left\{h \mid n_{\alpha}(h) \neq 0 \text { for some } \alpha\right\} .
\end{aligned}
$$

To expand around the fixed point we have to generalize some simple properties of power series to the infinite dimensional case:

Lemma 5.1. Let $f(\mathbf{x})$ be a formal power series in $\mathbf{x}=\left\{x_{\alpha}(h)\right\}_{\substack{h=0,1,2, \ldots \\ \alpha=1, \ldots, N}}$

$$
f(\mathbf{x})=\sum_{|\mathbf{n}| \geqq 1} f_{\mathbf{n}} \mathbf{x}^{\mathbf{n}}
$$

whose coefficients satisfy

$$
\left|f_{\mathbf{n}}\right| \leqq C^{|\mathbf{n}|+1} \varrho^{-h_{\max }(\mathbf{n})}, \quad C>0, \quad \varrho>1 .
$$

Then (5.4) is absolutely convergent for $\|\mathbf{x}\|_{\infty}<R \equiv C^{-1}\left(1-\varrho^{-1 / N}\right)$. Moreover, if $\|\mathbf{x}\|_{\infty} \leqq R_{1}<R$, the series

$$
g(\mathbf{y})=f(\mathbf{x}+\mathbf{y})=\sum_{\mathbf{n}} g_{\mathbf{n}}(\mathbf{x}) \mathbf{y}^{\mathbf{n}}
$$


is absolutely convergent for $\|\mathbf{y}\|_{\infty}<\tilde{R}\left(R_{1}\right)$ and

$$
\left|g_{\mathbf{n}}(\mathbf{x})\right| \leqq \widetilde{C}^{|\mathbf{n}|+1} \tilde{\varrho}^{-h_{\max }(\mathbf{n})}
$$

for some $\tilde{C}\left(R_{1}\right)>0, \varrho\left(R_{1}\right)>1$.

Proof. The first part of the lemma follows from

$$
\begin{aligned}
|f(\mathbf{x})| & \leqq \sum_{|\mathbf{n}| \geqq 1} C^{|\mathbf{n}|+1} \varrho^{-h_{\max }(\mathbf{n})}\|x\|^{|\mathbf{n}|}=\sum_{h} \varrho^{-h} \sum_{\mathbf{n}: h_{\max }(\mathbf{n})=h} C^{|\mathbf{n}|+1}\|x\|^{|\mathbf{n}|} \\
& \leqq C\|x\| \frac{1}{(1-C\|x\|)^{N}-\varrho^{-1}}<\infty,
\end{aligned}
$$

if $\|x\|<C^{-1}\left(1-\varrho^{-1 / N}\right)$. Similarly, using the explicit formula

we get the bound

$$
g_{\mathbf{m}}(\mathbf{x})=\sum_{\substack{\mathbf{n}: \\
n_{\alpha}(h) \geqq m_{\alpha}(h)}} f_{\mathbf{n}} \prod_{h, \alpha}\left(\begin{array}{c}
n_{\alpha}(h) \\
m_{\alpha}(h)
\end{array}\right) x_{\alpha}(h)^{n_{\alpha}(h)-m_{\alpha}(h)},
$$

$$
\left|g_{\mathbf{m}}(\mathbf{x})\right| \leqq \frac{C}{(1-C\|x\|)^{N}-\varrho^{-1}}(\varrho(1-C\|x\|))^{-h_{\max }(\mathbf{m})}\left(\frac{C}{1-C\|x\|}\right)^{|\mathbf{m}|},
$$

which proves (5.6), since, if $R_{1}<R, \varrho>1$,

$$
\tilde{\varrho} \equiv \varrho\left(1-C R_{1}\right)>\varrho^{1-1 / N} \geqq 1 .
$$

We can now write $\lambda_{\alpha}(k)=\lambda_{\alpha}^{*}+\xi_{\alpha}(k)$ and expand (5.1) in powers of $\xi_{\alpha}(k)$ by using Lemma 5.1 with $f=\beta_{\alpha}^{(0)}$ [this is sufficient since all $\beta_{\alpha}^{(k)}$ are equal to $\beta_{\alpha}^{(0)}$ up to scaling (3.10)]. We get

$$
\xi_{\alpha}(k)=\sum_{\beta} L_{\alpha \beta}^{(1)} \xi_{\beta}(k+1)+\varepsilon^{2} \sum_{\beta, h>k} L_{\alpha \beta k h}^{(2)} \xi_{\beta}(h)+[Q(\xi)]_{\alpha}(k),
$$

where $L^{(1)}$ is the linear part to first order in $\varepsilon$ [which couples only $\xi(k)$ to $\xi(k+1)$ ], $\varepsilon^{2} L^{(2)}$ is the remaining linear piece and $Q(\xi)$ is higher order in $\xi$. More precisely,

$$
L^{(1)}=\left(\begin{array}{ccc}
\gamma^{\varepsilon} & a \varepsilon & 0 \\
b \varepsilon & \gamma^{2-\varepsilon / 6} & c \varepsilon \\
d \varepsilon & f \varepsilon & \gamma^{-\varepsilon / 2}
\end{array}\right),
$$

where $a, b, \ldots, f$ are combinations of second order coefficients of the beta functional. For $\varepsilon$ (and thus $\lambda^{*}$ ) small,

$$
\begin{gathered}
\left|L_{\alpha \beta k h}^{(1)}\right| \leqq \text { const } \varrho^{-(h-k)}, \\
{[Q(\xi)]_{\alpha}(k)=\sum_{|\mathbf{n}| \geqq 2} Q_{\alpha \mathbf{n}}(k) \xi^{\mathbf{n}},} \\
\left|Q_{\alpha \mathbf{n}}(k)\right| \leqq C^{|\mathbf{n}|} \varrho^{-\left(h_{\max }(\mathbf{n})-k\right)}, \\
\left|Q_{\alpha \mathbf{n}}(k)\right|=0 \text { if } \quad h_{\min }(\mathbf{n})<k .
\end{gathered}
$$

By a linear transformation $\bar{\xi}_{\alpha}(k)=\sum_{\beta} A_{\alpha \beta} \xi_{\beta}(k)$, where $A_{\alpha \beta}(\varepsilon)$ is uniformly bounded in $a$, the matrix $L^{(1)}$ can be diagonalized to first order in $\varepsilon$ :

$$
\bar{\xi}=\bar{L}^{(1)} \bar{\xi}+\varepsilon^{2} \bar{L}^{(2)} \bar{\xi}+\bar{Q}(\bar{\xi}),
$$


where $\bar{L}^{(1)}=\operatorname{diag}\left(\gamma^{\varepsilon}, \gamma^{2-\varepsilon / 6}, \gamma^{-\varepsilon / 2}\right)$ and $\bar{L}^{(2)}, \bar{Q}$ satisfy the same bounds as $L^{(2)}, Q$, with different constants. The result of this section is the

Theorem 5.2. There exist constant $\varepsilon_{0}>0, r>0$ such that if $\varepsilon<\varepsilon_{0},\left|\bar{\xi}_{4}^{(0)}\right|<r,\left|\bar{\xi}_{2}^{(0)}\right|<r$, (5.14) has a solution $\left\{\bar{\xi}_{\alpha}(k)\right\}_{k=0, \alpha=4,2,2^{\prime}}^{\infty}$ with $\bar{\xi}_{\alpha}(0)=\bar{\xi}_{\alpha}^{(0)}, \alpha=4,2$, and $\bar{\xi}_{\alpha}(k) \rightarrow 0$ as $k \rightarrow \infty$ exponentially fast. For all $s$ with $0<s<\varepsilon, r=r(s)$ can be chosen so small that $\left|\bar{\xi}_{\alpha}(k)\right| \leqq r \gamma^{-s k}$.

By the transformation

$$
\lambda_{\alpha}(k)=\lambda_{\alpha}^{*}+\sum_{\beta}\left(A^{-1}\right)_{\alpha \beta} \bar{\xi}_{\beta}(k)
$$

this two-parameter family of solutions yields a two-parameter family of solutions of the flow equations which go asymptotically to the fixed point as $k \rightarrow \infty$, and a sequence of effective potentials $\left\{V^{(k)}\right\}_{k \geqq 0}$, solving thus the ultraviolet problem in the vicinity of the fixed point.

Proof. We use the contraction principle on the Banach space $\mathscr{B}_{s}$,

$$
\mathscr{B}_{s}=\left\{\boldsymbol{\xi}=\left\{\xi_{\alpha}(k)\right\}_{\alpha=4,2,2^{\prime}}\left|\|\boldsymbol{\xi}\|_{s} \equiv \sup _{k, \alpha}\right| \gamma^{s k} \xi_{\alpha}(k) \mid<\infty\right\},
$$

and write Eq. (5.14) with the initial condition $\xi_{\alpha}(0)=\xi_{\alpha}^{(0)}, \alpha=4,2$ (we drop the bars in the sequel), in the form of a fixed point equation:

where $T_{\xi(0)}(\xi)$ is defined by

$$
\xi=T_{\xi(0)}(\xi) \text {, }
$$

$$
\begin{aligned}
& {\left[T_{\xi(0)}(\xi)\right]_{4}(k)= \begin{cases}\gamma^{-\varepsilon}\left(\xi_{4}(k-1)-[S(\xi)]_{4}(k-1)\right), & k \geqq 1, \\
\xi_{4}^{(0)}, & k=0,\end{cases} } \\
& {\left[T_{\xi^{(0)}}(\xi)\right]_{2}(k)= \begin{cases}\gamma^{-(2-\varepsilon / 6)}\left(\xi_{2}(k-1)-[S(\xi)]_{2}(k-1)\right), & k \geqq 1, \\
\xi_{2}^{(0)}, & k=0,\end{cases} } \\
& {\left[T_{\xi^{(0)}}(\xi)\right]_{2^{\prime}}(k)=\gamma^{-\varepsilon / 2} \xi_{2^{\prime}}(k+1)+[S(\xi)]_{2^{\prime}}(k+1), \quad k \geqq 0,}
\end{aligned}
$$

where $S(\xi)=\varepsilon^{2} L^{(2)} \xi+Q(\xi)$.

Notice the different treatment of the irrelevant 2 -direction! Let $\mathscr{B}_{s}(r)$ $=\left\{\xi \in \mathscr{B}_{s} \mid\|\xi\|_{s} \leqq r\right\}$. For $\xi \in \mathscr{B}_{s}(r)$ and $\left|\xi_{\alpha}^{(0)}\right| \leqq r$,

$$
\left\|T_{\xi^{(0)}}(\xi)\right\|_{s} \leqq \max \left(\gamma^{-(\varepsilon-s)}, \gamma^{-\left(2-\frac{\varepsilon}{6}-s\right)}, \gamma^{-\left(\frac{\varepsilon}{2}+s\right.}\right) r+\varepsilon^{2} C^{\prime} r+C^{\prime \prime} r^{2} .
$$

$\left[C^{\prime}, C^{\prime \prime}\right.$, denote positive ( $\varepsilon$-independent) constants]. It follows that if $\varepsilon$ is small and $0<s<\varepsilon$, then $r$ can be chosen so small (depending on $s$ ) that

i.e. $\mathscr{B}_{s}(r)$ is mapped into itself.

$$
\left\|T_{\xi(0)}(\xi)\right\|_{s} \leqq r,
$$

Similarly, for $\xi, \boldsymbol{\eta} \in \mathscr{B}_{s}(r)$

$$
\begin{aligned}
\left\|T_{\xi^{(0)}}(\xi)-T_{\xi^{(0)}}(\boldsymbol{\eta})\right\|_{s} \leqq & \max \left(\gamma^{-(\varepsilon-s)}, \gamma^{-\left(2-\frac{\varepsilon}{6}-s\right)}, \gamma^{-\left(\frac{\varepsilon}{2}+s\right)}\right)\|\boldsymbol{\xi}-\boldsymbol{\eta}\|_{s} \\
& +C^{\prime} \varepsilon^{2}\|\boldsymbol{\xi}-\boldsymbol{\eta}\|_{s}+C^{\prime \prime \prime}\|\boldsymbol{\xi}-\boldsymbol{\eta}\|_{s^{r}} r
\end{aligned}
$$


where in estimating $|Q(\xi)-Q(\mathfrak{\eta})|$, use was made of

$$
\left\|\boldsymbol{\xi}^{\mathbf{n}}-\boldsymbol{\eta}^{\mathbf{n}}\right\|_{s} \leqq\|\boldsymbol{\xi}-\boldsymbol{\eta}\||\mathbf{n}| r^{|\mathbf{n}|-1}
$$

For $\varepsilon$ small, $0<s<\varepsilon, r$ small depending on $s$,

$$
\left\|T_{\xi(0)}(\boldsymbol{\xi})-T_{\xi(0)}(\boldsymbol{\eta})\right\|_{s} \leqq \theta\|\boldsymbol{\xi}-\boldsymbol{\eta}\|_{s}
$$

for some $\theta=\theta(\varepsilon, s)$ with $0<\theta<1$. The map $T_{\xi(0)}$ is thus a contraction on the closed subset $\mathscr{B}_{s}(r)$ of the Banach space $\mathscr{B}_{s}$. It follows that there exists a unique solution $\xi$ of (5.17) [or (5.14)] in $\mathscr{B}_{s}(r)$. This solution has the properties

$$
\begin{aligned}
\xi_{\alpha}(0) & =\xi_{\alpha}^{(0)}, \quad \alpha=4,2, \\
\left|\xi_{\alpha}(k)\right| & \leqq \gamma^{-s k} r .
\end{aligned}
$$

\section{Conclusions}

We have constructed a non-trivial fixed point of the renormalization group and a two-parameter family of theories whose ultraviolet behaviour is governed by this fixed point. This was done in the planar diagram approximation. One could extend this result to all orders in a $1 / N$ expansion of the matrix $\operatorname{tr} \phi^{4}$ model, since, at fixed order in $1 / N$, only Feynman diagrams with fixed Euler characteristic contribute. The number of such diagrams is bounded by const ${ }^{n}$ [8], with the same constant as in the planar case.

A very similar analysis can be made for the infrared problem for $\varepsilon<0$. This would give a rigorous understanding of the $\varepsilon$-expansion of critical exponents of statistical mechanics models in the planar approximation. We want, however, to point out that taking a propagator $1 / p^{2-\varepsilon / 2}$, rather than going to $4+\varepsilon$ dimension by means of some analytic continuation prescription, makes an important difference: in the model we studied in this paper there is no anomalous dimension at the non-trivial fixed point [see (4.7)]: the interacting field has the same dimension as the free field. To treat the problems with nontrivial anomalous dimension one has to modify slightly the formalism developed here, by introducing a rescaling at each renormalization step.

\section{Appendix. Solution of (4.4)}

The first equation in (4.4) can be divided by $\lambda_{4}^{*}$, since all graphs contributing to $\bar{\beta}_{4}$ have four external lines and must all have at least one quartic vertex. as

By rescaling $\hat{\lambda}_{4}=\bar{\varepsilon}^{-1} \lambda_{4}^{*}, \hat{\lambda}_{2}=\bar{\varepsilon}^{-2} \lambda_{2}^{*}, \hat{\lambda}_{2^{\prime}}=\bar{\varepsilon}^{-1} \lambda_{2}^{*}$, we can rewrite the equations

$$
\begin{aligned}
\hat{\lambda}_{4} & =-\beta_{444}^{-1}+\bar{\varepsilon} f_{4}\left(\hat{\lambda}_{4}, \hat{\lambda}_{2}, \hat{\lambda}_{2^{\prime}}\right), \\
\left(\gamma^{2}-1\right) \hat{\lambda}_{2} & =\beta_{244} \hat{\lambda}_{4}+\beta_{22^{\prime} 4} \hat{\lambda}_{2^{\prime}}, \hat{\lambda}_{4}+\bar{\varepsilon} f_{2}\left(\hat{\lambda}_{4}, \hat{\lambda}_{2}, \hat{\lambda}_{2^{\prime}}\right), \\
\hat{\lambda}_{2^{\prime}} & =\left(1+\gamma^{\varepsilon / 2}\right) \beta_{2^{\prime} 44} \hat{\lambda}_{4}^{2}+\bar{\varepsilon} f_{2^{\prime}}\left(\hat{\lambda}_{4}, \hat{\lambda}_{2}, \hat{\lambda}_{2^{\prime}}\right)
\end{aligned}
$$

for some functions $f_{4}, f_{2}, f_{2}$, analytic in $\left|\lambda_{\alpha}\right|<\frac{R}{\varepsilon}$ and uniformly bounded in $\varepsilon$ for $\left|\lambda_{\alpha}\right| \leqq R_{1}$, where $R_{1}$ is chosen large compared to the second order coefficients $\beta_{i j k}$. 
These equations can be easily brough to the form

$$
\hat{\lambda}_{\alpha}=\hat{\lambda}_{\alpha}^{(0)}+\bar{\varepsilon} \hat{f}_{\alpha}(\hat{\lambda})
$$

with, say, $\left|\hat{\lambda}_{\alpha}^{(0)}\right| \leqq \frac{R_{1}}{2}$; the implicit function theorem applies, and we get a solution of the form (4.5).

Acknowledgements. I would like to thank J. Fröhlich for illuminating comments and suggestions, and G. Gallavotti for teaching me the tree expansion and useful discussions. I gratefully acknowledge discussions with J. Imbrie, K. Gawedzki, and A. Kupiainen.

\section{References}

1. a) Gallavotti, G., Nicolò, F.: Renormalization theory in four dimensional scalar fields, I and II. Commun. Math. Phys. (in press, 1985)

b) Gallavotti, G.: Renormalization theory and ultraviolet stability for scalar fields via renormalization group methods. Rev. Mod. Phys. 57, 471-562 (1985)

c) Gallavotti, G.: The structure of renormalization theory: renormalization, form factors and resummations in scalar field theory. Lectures given at the 1984 Les Houches summer school "Critical phenomena, random systems, gauge theories"

2. a) Wilson, K.: Renormalization group and critical phenomena, I and II. Phys. Rev. B 4, 3174-3183 and 3184-3205 (1971)

b) Wilson, K., Kogut, J.: The renormalization group and the $\varepsilon$-expansion. Phys. Rep. 12, 75-200 (1974)

3. Polchinski, J.: Renormalization of effective Lagrangians. Nucl. Phys. B 231, 269-295 (1984)

4. Felder, G., Gallavotti, G.: Perturbation theory and non-renormalizable scalar fields. Commun. Math. Phys. (in press, 1985)

5. a) t'Hooft, G.: Rigorous construction of planar diagram field theories in four dimensional euclidean space. Commun. Math. Phys. 88, 1-26 (1983)

b) 't Hooft, G.: Planar diagram field theories. Lectures given at the 1983 Cargèse summer school "Progress in gauge field theories"

6. Rivasseau, V.: Construction and Borel summability of planar 4-dimensional euclidean field theory. Commun. Math. Phys. 95, 445-486 (1984)

7. Koplik, J., Neveu, A., Nussinov, S.: Some aspects of the planar perturbation series. Nucl. Phys. B 123, 109-131 (1977)

8. Bessis, D., Itzykson, C., Zuber, J.B.: Quantum field theory techniques in graphical enumeration. Adv. Appl. Math. 1, 109-157 (1980)

Communicated by K. Osterwalder

Received June 21, 1985 
